\title{
Quality Microeconomics
}

\author{
Jiancheng Liu \\ Dongfang Boiler Co., Ltd., Zigong, China \\ Email: decljc@163.com
}

Received 8 March 2016; accepted 23 March 2016; published 28 March 2016

Copyright (C) 2016 by author and OALib.

This work is licensed under the Creative Commons Attribution International License (CC BY).

http://creativecommons.org/licenses/by/4.0/

\section{(c) (i) Open Access}

\begin{abstract}
In traditional microeconomics, only the relationships between quantities and prices of products are taken into account, while the effects of products qualities on the prices, sales, production costs, utilization costs and environmental costs are scarcely or not considered. This paper discusses the differences and relations between the three parties of quality economics, quality economy analysis and quality economy, and sorts out the relevant research results in China and abroad. The paper analyzes the relationship among quality, price, demand and supply, introduces the value parameter, reconstructs the traditional formula for maximization of producer profit, and puts forward relevant policy suggestions: the government can supervise quality and environment by means of standards and taxation. With the stepwise perfection and increased strictness of standards, the quality level of a product will be higher and higher, and the environmental condition of a country or a region will be better and better. The quality level of a product is the most crucial factor affecting the producer profit and the social benefit.
\end{abstract}

\section{Keywords}

Quality Microeconomics, Product Value, Producer Profit, Utilization Cost, Environmental Cost, and Monitoring Game

Subject Areas: Economics

\section{1. 引言}

在传统经济学, 包括微观经济学、宏观经济学、经济增长和发展经济学以及环境经济学中, 很少或 根本就没有系统考虑产品质量对价格、销量、生产成本、使用成本、环境成本、国民收入以及经济发展 和增长等的影响。到目前为止, 还找不到任何一篇系统研究质量、环境和经济三者之间相互关系的正式 文献。实际上, 产品质量对价格、销量、生产成本、使用成本、环境成本、国民收入以及经济发展和增 长都有绝对的影响。 
什么是质量新经济学? 要回答这个问题, 首先让我们搞清楚质量经济学(Quality Economics)、质量 经济性分析(Quality Economic Analysis)和质量经济(Quality Economy)三者之间的区别和联系。

有学者认为质量经济学是一门从经济和经济学角度对质量问题进行系统研究的新兴经济学科 [1]。也 有学者认为: 质量经济学是一门研究质量问题在社会经济发展中的地位和作用、质量范畴所反映的社会 经济关系, 以及提高质量的社会经济条件的科学[2]。何晓晴[3]认为第一个定义可称为质量经济学的狭义 概念, 而第二个定义则可称为质量经济学的广义概念。狭义质量经济学研究的是质量的经济性问题, 有 时是具体的技术经济问题, 它多从企业角度来研究质量问题。广义质量经济学的研究对象, 是关于质量 的总体性经济理论问题, 而不是具体的技术经济问题, 它不是从企业内部而是从总体上来研究质量问题。

关于质量经济性分析的定义, 不同学者有不同看法。石培忠、谢承义[4]的说法是：质量经济分析就 是围绕产品(或服务)的适用性, 通过量本利的最优化分析, 以求得企业和社会最佳经济效益的一种技术经 济分析方法。研究为达到产品的质量水平而支付的费用与产品质量取得的经济效益是否相当, 乃是质量 经济分析的主要内容。银路、刘卫[5]认为: 所谓质量经济性分析, 就是对产品质量和质量管理进行经济 性分析和评价, 以期在提高产品质量的同时, 为企业创造最大的经济效益。何晓晴[3]则认为: 质量经济 性分析就是通过分析质量与经济之间的关系，探求成本最低、效益最高的经济性质量水平。

所谓质量经济, 深圳市质量发展战略课题组[6]的定义是: 以各个经济主体的知识的质量为主要动力 所创造出来的经济。就质量经济的针对性来分析, 从一般的实践意义上讲, 是针对着粗放条件下的数量 经济而言的; 从深层的理论意义上讲, 是针对着经典政治经济学的时间经济而言的。

何晓晴[3]认为: 要分清楚质量经济学、质量经济性分析和质量经济三者之间的关系, 就必须引入另 一个概念: 质量经济效益学。质量经济效益学(或质量经济效益分析)是一门专门研究质量与经济效益内在 联系和数量关系的学问或学科。质量经济学、质量经济效益学和质量经济性分析三者之间的关系为: 质 量经济学是研究质量经济的总学科, 质量经济效益学是质量经济学的一个分支和重要组成部分, 而质量 经济性分析则是质量经济效益学的一个分支和组成部分, 主要研究质量与企业经济效益之间的关系。至 于质量经济, 则是一种经济形态, 是着重产品质量的精细化条件下的经济。

在搞清楚质量经济学、质量经济性分析和质量经济三者之间的关系后, 让我们试着给质量新经济学 下个定义。笔者认为: 所谓质量新经济学, 就是同时从企业个体和社会总体两个层面系统研究产品质量 与社会经济和环境保护之间相互关系的学问或学科, 它是介于传统经济学、质量经济学和环境经济学三 者之间的一门交叉学科。质量新经济学也称为质量环境经济学。

\section{2。相关文献综述}

传统经济学[7]-[9]中, 生产厂商利润最大化分析没有考虑产品质量对价格、销量和生产成本的影响, 更没有考虑消费者的使用成本和环境成本。Vany 和 Saving [10]建立了一种一般竞争均衡条件可以得到满 足的质量决定模型, 它将获得产品所需的等待时间考虑为质量的外在形式。不过, 其分析结果却非常一 般化, 对具有特性 $\mathrm{z}$ 的所有产品都有效。在该模型中, 需求是价格和以 $\mathrm{z}$ 定义的一种度量的函数, 成本 是产量和该 $\mathrm{z}$ 度量的函数, $\mathrm{z}$ 是产量和生产能力的函数。预期的利润最大化厂商看起来就像垄断竞争者。 然而, 一旦给定不变的质量水平, 则可以获得完全竞争的结果。Sweeney [11]给出了一种当顾客面临质量 选择而非数量选择时商品的不证自明的特征化描述，推导了不同质量水平商品的个体特性和市场需求函 数, 同时演绎了一个或多个质量水平下相应于供给变化的比较静态价格变化特性, 并将该分析应用于住 宅市场中的价格变化。Dreze 和 Hagen [12]考察了价格的作用, 评价了市场机制在指导与产品差异化经济 体所生产产品的数量和质量同时相关的生产决策时的效率。Nancy [13]的研究发现支持一个宽泛的理论模 型, 建立了财务状况和产品质量之间的联系, 并且可能对安全检查和执法资源的分配具有重要的含义。 
Riley [14]、Plott 和 Miller [15]、Hassin [16]、Nevo [17]、Goolsbee 和 Petrin [18]等从信息经济学的角度研 究了产品质量问题。Jin 和 Kato [19]考察了互联网上拍卖中价格、质量、卖方主张和卖方声誉之间的联系。 Kumar [20]考察了相对于产品质量存在连续的买方需求强度, 并且垄断厂商与边际型买方进行交易而有利 可图时 (即存在短缺的情形), 而用商品垄断厂商实行的时间一致性临时价格 - 质量歧视。Biglaiser 和 Ma [21]考察了逆向选择情况下价格和质量竞争中的市场组织和效率问题。Yohei 和 Keita [22]研究了双寡头竞 争模式下广告与产品质量之间的动态关系。Brad [23]认为: 在垄断市场中, 产品质量信息的扩散可能取 决于产品质量信息是否是有利的。Kenneth [24]研究了产品质量信息的披露及其社会价值。Berndt、Bui、 Reiley 和 Urban [25]考察了美国抗溃疡药品行业成长和合并中市场营销、产品质量和价格竞争的作用。 Murphy [26]考察了美国地方服务行业中价格与质量的关系。Diehl、Kornish 和 Lynch [27]认为: 聪明的代 理人在较低的质量信息搜索成本下可以增加差异化和价格敏感度。研究质量 - 价格关系的文献较多, 本 文不再一一陈述。

在质量经济性研究方面, 费根堡姆(Feigenbuam)于 1956 年首先运用质量成本概念来分析问题。他在 《全面质量管理》(Total Quality Control) 一书中系统论述了质量成本概念, 并把质量成本划分为 “预防成 本”、“鉴定成本”、“内部故障成本” 和 “外部故障成本” 四个项目。在此基础上, 他建立了著名的 质量成本 - 质量水平模型。之后, 朱兰(Juran)和克罗斯比(Crosby)等也提出了类似的产品质量经济性和最 适宜质量水平模型[28]-[30]。

吴礼民[31]剖析了不同学者对质量经济性问题的不同看法, 界定了质量经济性概念, 阐明了对有关质 量经济性问题的基本观点, 揭示了质量成本的内涵和有关质量经济性的不同概念之间的联系。刘宏 [32] 通过综合考虑顾客满意、企业过程成本以及顾客和企业双方的利益, 从中寻找最佳结合点, 探讨和分析 企业如何有效实施质量经济性管理。银路[33]分析了不合格品率的经济性。王㑆、文昌俊[34]在制造企业 过程能力的基础上分析了过程能力的质量经济性, 给出了建立制造企业质量竞争力模型的方法, 并建立 了质量竞争力模型, 分析了模型参数, 得出了制造企业质量竞争力随参数的变化情况, 为制造企业质量 竞争力的形成机理研究提供了理论依据。魏国强[35]在经典的投入产出模型基础上, 引入质量成本、质量 追加投入等概念, 建立了多产品质量水平结构的投入产出模型, 并讨论了该模型在需求预测、计划以及 企业产品质量水平结构优化决策等方面的应用, 对传统质量经济分析方法和经典投入产出模型进行了推 广。强加成、徐景[36]论述了质量的经济性、质量经济分析、质量成本管理等问题, 阐明了质量成本管理 是统筹全局、做好全面质量管理工作强有力的方法和保障, 并提出了钢铁行业全面质量管理工作中一些 行之有效的具体做法。朱朝晖 [37]在传统质量成本理论的基础上提出了广义质量成本概念, 并分析了其特 性曲线。他认为, 质量成本分析必须考虑生产不良产品导致的间接损失, 包含间接质量损失的广义质量 成本总体上存在着不断下降的趋势, 高质量必然是低质量成本的。左春芳、白宝光[38], 黄培、汪蓉[39], 黄培、汪蓉、陈俊芳[40]在传统理论质量经济性分析方法的基础上, 结合对质量收入、质量成本和质量净 收益概念的界定，提出了基于质量净收益的质量经济性分析方法。赵建华、杨昌明、童庆英[41]在讨论传 统最优质量水平理论的基础上, 利用机会收益概念, 分析了追求卓越质量、获取质量竞争力进而获取机 会收益的一般原理, 提出垄断市场最佳质量水平的确定机制。

广义质量成本随着质量水平提高而不断下降, 当质量水平达到 $100 \%$ 合格时, 质量总成本最低, 这就 是 $6 \sigma$ 管理的理论基础。王金德、张东莉[42]和王金德、丁胜、张东莉[43]通过对质量经济性原理的研究, 阐述了质量与经济的关系以及如何通过提高顾客满意度和降低经营资源成本来实现质量经济性管理, 论 述了 $6 \sigma$ 管理与质量经济管理之间的关系(即质量经济性是 $6 \sigma$ 管理的基本原则), 并结合 $6 \sigma$ 管理理论和方 法的实践, 描述了 $6 \sigma$ 管理就是致力于把质量和财务业绩联系起来的新概念, 提出了 $6 \sigma$ 三部曲: $6 \sigma$ 组织 
(OFSS)、6 $\sigma$ 策划(DFSS)和 $6 \sigma$ 过程(PFSS)的观点。王金德[44]通过对 6 $\sigma$ 质量及其管理理论的研究, 论述了 $6 \sigma$ 质量与经济性的关系, 提出 $6 \sigma$ 管理以经济性为核心致力于把质量与财务业绩联系起来的观点, 通过开

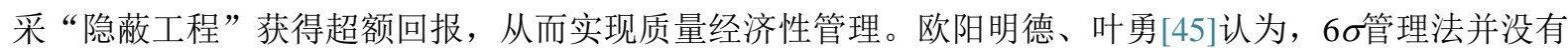
对传统质量管理方法完全加以摒弃, 而是在沿袭现代质量管理理论和方法的基础上的一种发展。他们着 重对 $6 \sigma$ 管理法的质量经济性进行了分析。

质量经济性分析的上述文献基本上都是建立在一般或广义质量成本-质量水平模型基础上的, 其局限 性在于: 未能将产品质量水平与市场绩效联系起来。Bajic [46]认为, 产品的质量水平会对产品的效用、 价格、销量和市场竞争力等产生影响, 并最终影响其获利性。张卓[47]以企业利润最大化为目标, 引入效 用概念, 提出了一个基于市场的最适宜质量水平模型。该模型突破了传统质量经济学理论仅以质量成本 确定最适宜质量水平的模式, 将质量水平与产品成本、效用和市场绩效联系起来, 使模型具有更为广泛 的适用性。他还就市场不确定性、消费者偏好、技术进步、市场竞争等因素对模型的影响展开了讨论, 揭示了质量、销量、价格、成本以及技术进步和竞争之间的关系, 为企业产品决策、技术改造决策和质 量策划提供了一种参考。曾凤章、崔丽[48]从顾客价值角度对产品质量与产品寿命周期费用的关系、产品 质量价格关系进行了分析, 从而得出产品质量对顾客价值的影响; 同时从企业角度考虑产品质量对产品 制造成本的影响, 以此为基础建立了基于顾客价值的质量经济性模型, 并在分析过程中提出了持续改进 产品质量、相对折价等提升顾客价值的途径。陈江彪、张卓[49]比较了企业从自身出发和以顾客满意为焦 点制定质量水平策略的差异, 提出了基于顾客满意的适宜质量水平模型, 得出企业以自身为焦点所制定 的适宜质量水平逊于以顾客满意为焦点的适宜质量水平; 指出产品质量成本随产品合率的改善而降低, 并通过引入产品合格率弹性系数分析了质量水平提高对企业利润的影响。

从质量经济效益学(质量经济效益分析)层面进行研究的文献不多, 笔者只查到了解文锋、银路[50] (进 行了交货期的质量经济分析), 翟瑞华[51] (对企业质量经济效益的控制进行了实证研究), 关晓光、葛志 杰[52](研究了质量经济效益的模糊综合评价问题), 李少华[53] (研究了质量经济效益分析问题)等几篇。

王兵才[54]对质量经济学的研究对象、任务及特点以及质量经济学研究的内容进行了探索性研究。郭 克莎[2]则进行了开创性的系统研究。李江帆[55]认为, 郭克莎的研究以较为深刻的理论分析和自成一派 的逻辑结构, 构建了质量经济学这一门新学科的理论体系; 它重视理论创新, 以质量两重性为理论基础, 提出和分析了关于质量经济学的一系列颇具新意的范畴和原理; 它立足中国实践, 重点抓宏观分析, 揭 示了产品质量与经济增长和发展的关系, 以及提高产品质量的社会经济条件, 为搞好我国质量管理提供 了有价值的思路和对策。银路 [1]介绍了质量经济思想的形成和发展以及质量经济学的研究目的、内容、 思路和方法, 评述了质量经济学的发展现状。张卓[56]认为: 质量既是企业获取和保持竞争优势的重要因 素, 又是构成企业经济的基本组成要素; 有效的质量活动必须以经济性为基础, 一方面为顾客增加可感 知的价值, 另一方面为企业提高收益和持续竞争力。他分析了质量成本理论、质量投入和产出理论, 以 及基于知识的质量经济学的发展情况及其对质量活动的指导意义, 并进一步探索了质量经济学研究的发 展方向。

在传统宏观经济学 [7] [9] [57]-[59]和经济增长和发展经济学[60]-[64]中, 基本没有讨论产品质量问题, 也没有考虑产品质量对国民收入和经济发展及增长的影响。

环境经济学[65]介绍了经济系统与环境系统之间的关系, 阐述了供需原理和经济效率问题, 重点进行 了环境的收益-成本分析, 并论述了环境政策设计的原则, 探讨了国际环境方面的问题。Kohn [66]利用 线性规划模型研究了美国 St. Louis 地区的最优空气质量标准问题。王学军[67]讨论了环境质量经济价值 度量的若干问题。黄淑芹、王丽[68]从博弯论的角度建立数学模型以分析环境恶化的原因: 公共环境的性 质及企业追求利益最大化、个体理性导致自然资源被过度使用而破坏环境；对于在环境治理上出现的问 
题, 设计了一个完全信息静态博弯, 用以分析企业治理和部门监督之间的联系, 推出如何有效地减少监 督和治理成本。然而, 目前环境经济学相关的理论主要研究的是生产过程中的环境污染及其事后治理, 而未涉及环境问题的根源一一产生污染的各种产品的环境性能(产品质量)和人们的不良行为。

笔者通过互联网及专业机构检索, 没有发现任何有关质量环境经济学的正式文献, 近似文献只有一 篇, 即胡品洁[69]。胡品洁认为: 随着生产力水平的进一步提高和环境的恶化, 在质量经济分析中应该引 入两种新视角: 顾客导向和环境保护; 这些观念可以在质量成本、质量经济效益和质量改进中得到贯彻; 但为了落实这些观念, 必须给企业一定的压力或刺激。胡品洁的研究虽有一定价值, 然而却过于粗䊁和 缺乏系统性。

总之, 在传统经济学, 包括微观经济学(含质量经济学、质量经济性分析和质量经济效益学)、宏观经 济学、经济增长和发展经济学以及环境经济学中, 很少或根本就没有系统考虑产品质量对价格、销量、 生产成本、使用成本、环境成本、国民收入以及经济发展和增长等的影响。到目前为止, 还找不到任何 一篇系统研究质量、环境和经济三者之间相互关系的正式文献。而本文一一质量微观经济学, 旨在从企 业个体和政府两个层面系统研究产品质量与市场经济和环境保护之间的相互关系, 将传统经济学、质量 经济学和环境经济学三者有机地联系起来, 深入分析产品质量对价格、销量、生产成本、使用成本和环 境成本等的影响, 奠定质量新经济学——质量环境经济学的微观基础。

\section{3. 质量微观经济学}

\section{（一）质量、价格、需求和供给之间的关系}

在传统微观经济学理论中, 一般认为需求主要与价格相关, 是价格的减函数, 即 $D=D(P), \frac{\mathrm{d} D}{\mathrm{~d} P}<0$, 供给也主要与价格相关, 是价格的增函数, 即 $S=S(P), \frac{\mathrm{d} S}{\mathrm{~d} P}>0$, 而不考虑质量对价格、需求和供给的 影响。实际上, 任何商品的需求是其性价比 $V$ 的增函数, 即 $D=D(V), \frac{\mathrm{d} D}{\mathrm{~d} V}>0$, 而任何商品的供给是 其性价比 $V$ 的减函数, 即 $S=S(V), \frac{\mathrm{d} S}{\mathrm{~d} V}<0$ 。图 1 所示为上述关系的比较。

$V$ 是质量 $q$ 和价格 $P$ 的函数, 有:

$$
V=\frac{q \cdot P_{0}}{P}
$$

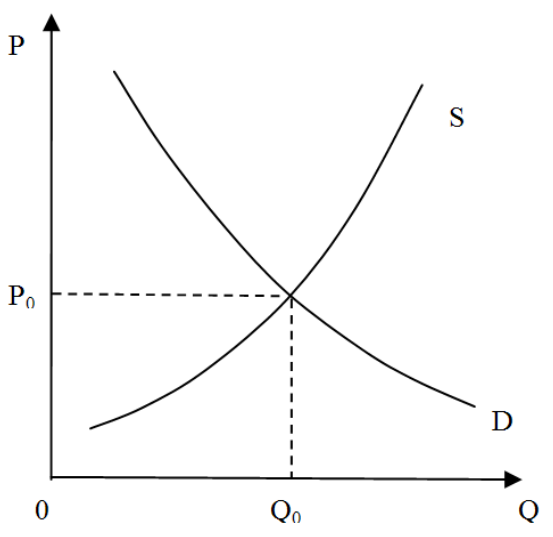

(a)

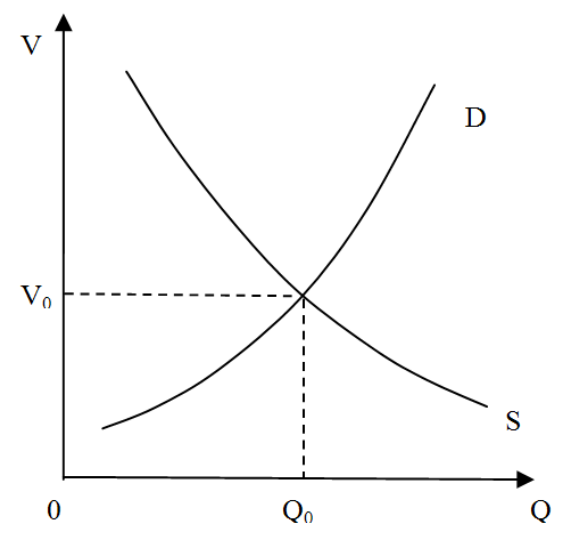

(b)

图 1. 传统供需关系(a)和刘氏供需关系(b)的比较. 
$P_{0}$ 为合格商品的平均价格。

实际上, $V$ 即商品的价值系数, 供需平衡时商品的产量是 $V$ 的函数。有:

$$
Q=Q(V)
$$

参数 $q$ 的特性如下:

当 $q$ 为产品的符合性质量时, $q=0-100 \%$; 当 $q$ 为产品的质量水平时, $q \geq 1$ 。

对生产要素的需求和供给也可以进行类似的分析。

厂商可以生产和销售价格和质量水平差异显著而价值系数相当的系列产品, 以使自己的市场份额最 大化。考虑公式(1), 该厂商的总产出如下:

$$
y_{P}=\sum Q_{i} \cdot P_{i}=\sum Q_{i} \cdot \frac{q_{i} \cdot P_{0}}{V}=\frac{1}{V} \sum Q_{i} \cdot q_{i} \cdot P_{0}
$$

公式(3)非常重要, 将在本人以后的研究中广泛应用。

对顾客来说, 当 $V<1$, 他会对产品不太满意并感到价格太高和不公平; 当 $V=1$, 他会对产品基本 满意并觉得价格是公平的; 当 $V>1$, 他会对产品比较满意并觉得价格便宜和更加公平。一句话, 顾客会 考虑购买该产品是否划算。对厂商来说, 当 $V>1$, 他会觉得价格太便宜、不公平和不太满意; 当 $V=1$, 他会觉得价格公平并基本满意: 当 $V<1$, 他会觉得价格更公平并更加满意。一句话, 厂商会考虑出售其 产品是否划算。经过顾客和厂商之间的反复博弯, $V$ 将趋向于等于 1 。对整个社会来讲, 竞争信息基本 完备, 我们可以取 $V=1$ 。

\section{(二) 传统厂商利润公式}

在传统经济学[7]中, 对厂商利润最大化的其表述如下:

厂商生产的目的是为了追求最大的利润。在完全竞争条件下, 对厂商来说, 商品的价格和生产要素 的价格都是既定的, 厂商可以通过对生产要素投入量的不断调整来实现最大利润。厂商在追求最大利润 的过程中, 可以得到最优的要素组合。

假定：在完全竞争条件下，企业的生产函数为 $Q=f(L, K)$, 既定的商品的价格为 $P$, 既定的劳动的 价格和资本的价格分别为 $w$ 和 $r, \pi$ 表示利润。由于厂商的利润等于收益减成本，于是厂商的利润函数 为:

$$
\pi(L, K)=P \cdot f(L, K)-(w L+r K)
$$

式中, $P \cdot f(L, K)$ 表示收益, $(w L+r K)$ 表示成本。

利润最大化的一阶条件为:

$$
\begin{aligned}
& \frac{\partial \pi}{\partial L}=P \frac{\partial f}{\partial L}-w=0 \\
& \frac{\partial \pi}{\partial K}=P \frac{\partial f}{\partial K}-r=0
\end{aligned}
$$

根据以上两式，可以得到:

$$
\frac{\frac{\partial f}{\partial L}}{\frac{\partial f}{\partial K}}=\frac{M P_{L}}{M P_{K}}=\frac{w}{r}
$$

式中, $M P_{L}$ 和 $M P_{K}$ 分别为劳动和资本的边际产量。该式表明, 追求利润最大化的厂商可以得到最优的生 产要素组合。 


\section{(三) 厂商利润公式的重建}

\section{1. 产品的使用成本和环境成本}

由于质量水平的不同, 产品的使用成本和环境成本是有较大差异的。产品的使用成本包括使用过程 中消耗的能源、水资源等的费用、产品维护费用和产品寿命到期后的处理费用。产品的环境成本包括产 品生产过程中的环境成本和产品使用过程中的环境成本。产品使用过程中的环境成本包括其使用过程中 以及寿命到期后的处理过程中产生的环境污染成本。

国家应在各种产品的设计制造标准中规定与使用成本和使用过程中的环境成本相关的指标，如能耗 指标、水耗指标、可靠性指标、寿命指标和污染排放指标等, 生产厂商的产品必须达到标准要求才允许 出厂销售。实际上, 这些指标皆与产品的质量相关, 可统称为质量指标。生产厂商必须在产品说明书中 出具产品能耗指标、水耗指标、可靠性指标、寿命指标和污染排放指标等的相应数据; 同时, 国家应对 产品质量进行监督，并及时向公众发布有关信息。消费者将在比较充分的信息条件下对生产厂商的产品 进行选择购买。对违规出售未达标产品的生产厂商, 国家将没收其全部不合格产品, 并处以罚款; 对实 际指标特别优异的生产厂商, 国家应给予奖励。

对于生产过程中的环境成本, 它与产品质量关系不大, 而与生产工艺和产量密切相关。国家应就各 种产品的生产过程制定环境污染排放标准，生产厂商的产品生产过程必须达到标准要求才允许进行。国 家应对产品生产过程中的污染排放指标进行监督, 对未达标生产厂商处以罚款, 对实际指标特别优异的 生产厂商给予奖励。

\section{2. 不考虑生产过程中的环境成本时厂商利润的最大化}

为简化问题的分析, 设想厂商只生产和销售一种产品(多种产品的情况可利用矩阵进行分析, 本文不 作讨论。)且先暂不考虑生产过程中的环境成本。

生产成本 $C$ 是产量和性价比的函数, 有 $C=C(Q, V)$ 和 $Q=f(L, K)$ 。

下面分三种情况进行分析。

\section{(1) 生产厂商质量达标}

如生产厂商生产的产品, 其能耗指标、水耗指标、可靠性指标、寿命指标和污染排放指标等，均符 合国家标准要求, 则有:

$$
\pi(P, V)=P \cdot Q(V)-C(Q(V), V)
$$

利润最大化的一阶条件为:

$$
\begin{gathered}
\frac{\partial \pi}{\partial P}=Q(V)+P \cdot \frac{\mathrm{d} Q}{\mathrm{~d} V} \cdot \frac{\mathrm{d} V}{\mathrm{~d} P}-\left(\frac{\partial C}{\partial Q} \cdot \frac{\mathrm{d} Q}{\mathrm{~d} V} \cdot \frac{\mathrm{d} V}{\mathrm{~d} P}+\frac{\partial C}{\partial V} \cdot \frac{\mathrm{d} V}{\mathrm{~d} P}\right)=0 \\
\frac{\partial \pi}{\partial V}=P \cdot \frac{\mathrm{d} Q}{\mathrm{~d} V}-\left(\frac{\partial C}{\partial Q} \cdot \frac{\mathrm{d} Q}{\mathrm{~d} V}+\frac{\partial C}{\partial V}\right)=0
\end{gathered}
$$

联立求解(7)、(8)二式, 可以求出质量达标厂商的最佳产品价格和最佳性价比(产品质量水平)。此外, 公式(5)仍然成立, 即追求利润最大化的厂商可以得到最优的生产要素组合。问题得解。

\section{(2) 生产厂商质量优异}

如生产厂商生产的产品, 其能耗指标、水耗指标、可靠性指标、寿命指标和污染排放指标等, 不仅 均符合国家标准要求, 而且特别优异, 则国家可以考虑对其进行奖励。设奖励金额 $B_{1}$ 与产品销量成正比, $B_{1}=b_{1}(V) \cdot Q(V)\left(b_{1}(V)\right.$ 为单位产品奖金额，与性价比(质量水平)相关。)。那么有:

$$
\pi(P, V)=P \cdot Q(V)-C(Q(V), V)+b_{1}(V) \cdot Q(V)
$$


利润最大化的一阶条件为:

$$
\begin{aligned}
\frac{\partial \pi}{\partial P}= & Q(V)+P \cdot \frac{\mathrm{d} Q}{\mathrm{~d} V} \cdot \frac{\mathrm{d} V}{\mathrm{~d} P}-\left(\frac{\partial C}{\partial Q} \cdot \frac{\mathrm{d} Q}{\mathrm{~d} V} \cdot \frac{\mathrm{d} V}{\mathrm{~d} P}+\frac{\partial C}{\partial V} \cdot \frac{\mathrm{d} V}{\mathrm{~d} P}\right) \\
& +\left(\frac{\mathrm{d} b_{1}}{\mathrm{~d} V} \cdot \frac{\mathrm{d} V}{\mathrm{~d} P} \cdot Q+b_{1} \cdot \frac{\mathrm{d} Q}{\mathrm{~d} V} \cdot \frac{\mathrm{d} V}{\mathrm{~d} P}\right)=0 \\
\frac{\partial \pi}{\partial V}= & P \cdot \frac{\mathrm{d} Q}{\mathrm{~d} V}-\left(\frac{\partial C}{\partial Q} \cdot \frac{\mathrm{d} Q}{\mathrm{~d} V}+\frac{\partial C}{\partial V}\right)+\left(\frac{\mathrm{d} b_{1}}{\mathrm{~d} V} \cdot Q+b_{1} \cdot \frac{\mathrm{d} Q}{\mathrm{~d} V}\right)=0
\end{aligned}
$$

联立求解(10)、(11)二式, 可以求出质量优异厂商的最佳产品价格和最佳性价比(产品质量水平)。此 外, 有: $\pi(L, K)=\left(P+b_{1}\right) \cdot f(L, K)-(w L+r K)$ 。我们也可以得到公式(5), 即, 追求利润最大化的厂商 可以得到最优的生产要素组合。问题得解。

\section{(3) 生产厂商质量不达标}

如生产厂商生产的产品, 其能耗指标、水耗指标、可靠性指标、寿命指标和污染排放指标等主要质 量指标中, 一项或多项不符合国家标准要求, 则产品不允许销售。对违规出售未达标产品的生产厂商, 国家将没收其全部不合格产品, 并处以罚款, 同时勒令其限期整改, 只有当其产品完全达到标准要求后, 才允许继续出厂销售。

张维迎 $[70]$ 讨论了监督博亦问题, 生产厂商质量不达标的情况与此类似。博亦的参与人为监督机关和 生产厂商, 监督机关的纯战略选择是检查或不检查, 生产厂商的纯战略选择是违规生产销售或达标生产 销售。表 1 概括了对应不同纯战略组合的支付矩阵。其中, $C_{G 1}$ 为监督机关的检查成本, $F_{1}$ 是罚款金额, $\pi_{l 1}$ 为厂商合法生产销售时的利润, $\pi_{u 1}$ 为厂商违规生产销售被发现时的利润, $\pi_{u 2}$ 为厂商违规生产销售未 被发现时的利润。设 $C_{E 1}$ 为生产厂商合法生产销售时, 相对于违规生产销售时所增加的成本, 即整改成 本, 则有 $C_{E 1}=\pi_{u 2}-\pi_{l 1}$ 。 $\pi_{l 1}$ 的表达式如(5)式所示, $\pi_{u 1}$ 的表达式如下:

$$
\pi_{u 1}=P \cdot q \cdot Q(V)-C(Q(V), V)
$$

假定 $C_{G 1}<F_{1}$, 此时不存在纯战略纳什均衡, 但可以求解混合战略纳什均衡。设监督机关检查的概率 为 $\theta$, 生产厂商违规生产销售的概率为 $\gamma$ 。给定 $\gamma$, 监督机关选择检查 $(\theta=1)$ 和不检查 $(\theta=0)$ 的期望收 益分别为:

$$
\begin{gathered}
\pi_{G 1}(1, \gamma)=\left(F_{1}-C_{G 1}\right) \gamma+\left(-C_{G 1}\right)(1-\gamma)=\gamma F_{1}-C_{G 1} \\
\pi_{G 1}(0, \gamma)=0 \gamma+0(1-\gamma)=0
\end{gathered}
$$

\begin{tabular}{|c|c|c|c|}
\hline & & \multicolumn{2}{|c|}{ 生产厂商 } \\
\hline & & 合法生产销售 & 违规生产销售 \\
\hline \multirow{2}{*}{ 监督机关 } & 检查 & $-C_{G 1}, \quad \pi_{l 1}$ & $F_{1}-C_{G 1}, \quad \pi_{u 1}-F_{1}$ \\
\hline & 不检查 & $0, \quad \pi_{l 1}$ & $0, \quad \pi_{u 2}=\pi_{l 1}+C_{E 1}$ \\
\hline
\end{tabular}

解 $\pi_{G 1}(1, \gamma)=\pi_{G 1}(0, \gamma)$, 得: $\gamma^{*}=\frac{C_{G 1}}{F_{1}}$ 。即: 如果生产厂商违规生产销售的概率小于 $C_{G 1} / F_{1}$, 监督 机关的最优选择是不检查; 如果生产厂商违规生产销售的概率大于 $C_{G 1} / F_{1}$, 监督机关的最优选择是检查; 如果生产厂商违规生产销售的概率等于 $C_{G 1} / F_{1}$, 监督机关随机地选择检查或不检查。

给定 $\theta$, 生产厂商选择合法生产销售 $(\gamma=0)$ 和违规生产销售 $(\gamma=1)$ 的期望收益分别为:

\section{表 1. 监督机关与生产厂商的博弯}




$$
\begin{gathered}
\pi_{E 1}(\theta, 0)=\pi_{l 1} \theta+\pi_{l 1}(1-\theta)=\pi_{l 1} \\
\pi_{E 1}(\theta, 1)=\left(\pi_{u 1}-F_{1}\right) \theta+\left(\pi_{l 1}+C_{E 1}\right)(1-\theta)=\left(\pi_{l 1}+C_{E 1}\right)-\left(\pi_{l 1}-\pi_{u 1}+C_{E 1}+F_{1}\right) \theta
\end{gathered}
$$

解 $\pi_{E 1}(\theta, 0)=\pi_{E 1}(\theta, 1)$, 得: $\theta^{*}=\frac{C_{E 1}}{\pi_{l 1}-\pi_{u 1}+C_{E 1}+F_{1}}$ 。即: 如果监督机关检查的概率小于 $C_{E 1} /\left(\pi_{l 1}-\pi_{u 1}+C_{E 1}+F_{1}\right)$, 生产厂商的最优选择是违规生产销售; 如果监督机关检查的概率大于 $C_{E 1} /\left(\pi_{l 1}-\pi_{u 1}+C_{E 1}+F_{1}\right)$, 生产厂商的最优选择是合法生产销售; 如果监督机关检查的概率等于 $C_{E 1} /\left(\pi_{l 1}-\pi_{u 1}+C_{E 1}+F_{1}\right)$, 生产厂商随机地选择合法生产销售或违规生产销售。

因此, 混合战略纳什均衡是: $\theta^{*}=C_{E 1} /\left(\pi_{l 1}-\pi_{u 1}+C_{E 1}+F_{1}\right), \gamma^{*}=C_{G 1} / F_{1}$, 即监督机关以 $C_{E 1} /\left(\pi_{l 1}-\pi_{u 1}+C_{E 1}+F_{1}\right)$ 的概率检查, 生产厂商以 $C_{G 1} / F_{1}$ 的概率选择违规生产销售。这个均衡的另一个 可能的 (或许更为合理的) 解释是, 经济中有许多个生产厂商, 其中有 $C_{G 1} / F_{1}$ 比例的生产厂商选择违规 生产销售, $\left(1-C_{G 1} / F_{1}\right)$ 比例的生产厂商选择合法生产销售; 监督机关随机地检查 $C_{E 1} /\left(\pi_{l 1}-\pi_{u 1}+C_{E 1}+F_{1}\right)$ 比例的生产厂商的生产销售情况。

监督博弯的纳什均衡与监督机关的检查成本 $C_{G 1}$ 、生产厂商合法生产销售时所增加的成本 $C_{E 1}$ 、罚款 金额 $F_{1}$ 、以及厂商合法生产销售时的利润 $\pi_{l 1}$ 和厂商违规生产销售被发现时的利润 $\pi_{u 1}$ 有关。对违规生产 销售的惩罚越重、监督机关的检查成本越低, 厂商选择违规生产销售的概率越小; 对违规生产销售的惩 罚越轻、监督机关的检查成本越高, 厂商选择违规生产销售的概率越大。这个结论与我们关于生产厂商 逃避检查的技术和检查成本的假设有关。我们假定一旦监督机关检查, 违规生产销售就会被发现。如果 不是这样, 比如说, 如果违规严重而获利颇多的生产厂商有更好的办法进行隐蔽生产销售, 从而使违规 行为更难被发现, 这个结论就不一定成立了。此外, 违规严重而获利颇多的生产厂商可能更有积极性贿 赂监督官员, 在这种情况下, 上述结论也难以成立。但是有一点可以肯定的是, 通过提高对违规者的惩 罚, 生产厂商违规的积极性就会下降, 监督机关检查的必要性也会降低。所以, 监督机关应努力改进检 查技术, 降低检查成本, 并应制定科学的罚款制度, 比如根据违规产品的销量和违规性质(产品合格率的 高低、有无造成严重后果和不良影响等)决定罚款额度。一般情况下, 合格率越高、违规后果越轻、影响 越小, 罚款越轻; 合格率越低、违规后果越严重、影响越大, 罚款越重。这样, 违规性质越严重, 出现 的可能性越小, 即严重违规厂商的数量和严重违规的次数越少。

下面详细分析一下 $\theta^{*}=C_{E 1} /\left(\pi_{l 1}-\pi_{u 1}+C_{E 1}+F_{1}\right)$ 的意义。由于 $C_{E 1}>0, \pi_{l 1}-\pi_{u 1}>0, F_{1}>0$, 所以 $0<\theta^{*}<1$ 。根据广义质量成本理论, 一般情况下, 生产厂商违规性质越严重, $\left(\pi_{l 1}-\pi_{u 1}\right)$ 的值就越大, $C_{E 1}$ 的值也越大。假设 $\left(\pi_{l 1}-\pi_{u 1}\right)$ 的值与 $C_{E 1}$ 的值成正比, 那么, 如果 $F_{1}$ 给定, 则违规性质越严重, $\theta^{*}$ 的值越 大, 即监督机关需要对有严重违规倾向的生产厂商加大检查力度。但是, 如果设定 $F_{1}$ 的值与 $\left(\pi_{l 1}-\pi_{u 1}\right)$ 和 $C_{E 1}$ 的值成正比, 则不管违规性质如何, $\theta^{*}$ 的值可以基本保持不变, 有 $\theta^{*} \cong \theta_{0}^{*}$ 。所以, 如果罚款额度 $F_{1}$ 设置合理, 只要监督机关以 $\theta_{0}^{*}$ 以上的概率检查所有生产厂商的生产销售情况, 或随机地检查 $\theta_{0}^{*}$ 以上比例 生产厂商的生产销售情况, 则生产厂商选择违规生产销售的期望收益将小于选择合法生产销售的期望收 益, 那么, 生产厂商在大多数情况下将选择合法生产销售, 或者说, 一般情况下, 大多数的生产厂商将 选择合法生产销售, 结果理想时, 则可以基本杜绝违规生产销售情况的发生。 $F_{1}$ 的设置可以如下: $F_{1}=f_{1}(q) \cdot Q(V)$, 其中, $f_{1}(q)$ 为单位产品罚款金额, 是质量 $q$ (以产品合格率表示)的函数, 且与 $q$ 成反 比例关系, $q$ 越高罚款越轻, $q$ 越低罚款越重; $Q(V)$ 为产品销量。

就质量不达标生产厂商的竞争力而言, 当监督机关的检查概率小于 $\theta^{*}=C_{E 1} /\left(\pi_{l 1}-\pi_{u 1}+C_{E 1}+F_{1}\right)$ 时, 其竞争力强于质量达标生产厂商; 当监督机关的检查概率大于 $\theta^{*}=C_{E 1} /\left(\pi_{l 1}-\pi_{u 1}+C_{E 1}+F_{1}\right)$ 时, 其竞争力 弱于质量达标生产厂商; 当监督机关的检查概率等于 $\theta^{*}=C_{E 1} /\left(\pi_{11}-\pi_{u 1}+C_{E 1}+F_{1}\right)$ 时, 其竞争力与质量达 
标生产厂商的竞争力相同。由于质量不达标生产厂商的违规生产销售行为会损害消费者的利益, 并有可 能产生严重的后果和恶劣的影响, 所以, 监督机关必须以大于 $\theta^{*}=C_{E 1} /\left(\pi_{l 1}-\pi_{u 1}+C_{E 1}+F_{1}\right)$ 的概率对生产 厂商的生产销售情况进行检查, 以减少或杜绝违规生产销售情况的发生。监督机关应及时准确地公布检 查结果, 让公众了解有关产品的质量信息, 科学合理地做出消费选择, 使质量不达标产品根本就没有生 存的空间和机会。

由于没有积极意义, 此处不再求解质量不达标厂商的最佳劳动投入、最佳资本投入、最佳产品价格 和最佳产品质量。

\section{(四) 考虑生产过程中的环境成本时厂商利润的最大化}

如对生产过程中的环境成本进行量化, 设其为产量和生产工艺参数的函数, 那么有:

$$
C_{3}(Q, T)=C_{3}(T) \cdot Q(V)
$$

其中, $T$ 为生产工艺参数; $c_{3}(T)$ 为单位产品生产过程中的环境成本, 与工艺水平 $T$ 相关。

国家应就各种产品的生产过程制定环境污染排放标准, 生产厂商的产品生产过程必须达到标准要求 才允许进行。对环保达标生产厂商, 国家不征收任何款项; 对环保未达标生产厂商, 国家应征收环境税 或处以罚款；对生产环保特别优异的厂商，国家应给予奖励。

国家应根据厂商生产过程中环境成本的大小进行征税、处以罚款或实施奖励。

将生产厂商环保是否达标与所生产产品的质量情况同时考虑, 则存在 9 种情形(如表 2 所示)。

下面重点分析三种情形: (1) 质量达标, 环保达标; (2) 质量优异, 环保优异; (3) 质量达标, 环保 不达标。至于其它情形, 可做类似的分析。质量、环保同时不达标时, 政府应勒令企业停产整顿, 本文 不做进一步讨论。

\section{1. 质量达标, 环保达标}

该情形的分析与三.(三).2.(1)的分析完全相同，在此不再赘述。

\section{2. 质量优异, 环保优异}

在该情形下, 国家不仅对生产厂商的产品质量进行奖励, 而且对其生产方法也进行奖励。对产品质 量的奖励为 $B_{1}$ (见三. (三).2.(2)), 对生产方法的奖励为 $B_{2}$ 。设奖励金额 $B_{2}$ 也与产品销量成正比, $B_{2}=b_{2}(T) \cdot Q(V)$ ( $b_{2}(T)$ 为单位产品奖金额, 与工艺 $T$ 水平相关。此时, 生产成本是产量、性价比(质 量水平 $)$ 和工艺水平的函数, $C=C(Q, V, T)$ 。那么有:

$$
\pi(P, V, T)=P \cdot Q(V)-C(Q, V, T)+b_{1}(V) \cdot Q(V)+b_{2}(T) \cdot Q(V)
$$

利润最大化的一阶条件为:

$$
\begin{aligned}
\frac{\partial \pi}{\partial P}= & Q(V)+P \cdot \frac{\mathrm{d} Q}{\mathrm{~d} V} \cdot \frac{\mathrm{d} V}{\mathrm{~d} P}-\left(\frac{\partial C}{\partial Q} \cdot \frac{\mathrm{d} Q}{\mathrm{~d} V} \cdot \frac{\mathrm{d} V}{\mathrm{~d} P}+\frac{\partial C}{\partial V} \cdot \frac{\mathrm{d} V}{\mathrm{~d} P}\right) \\
& +\left(\frac{\mathrm{d} b_{1}}{\mathrm{~d} V} \cdot \frac{\mathrm{d} V}{\mathrm{~d} P} \cdot Q+b_{1} \cdot \frac{\mathrm{d} Q}{\mathrm{~d} V} \cdot \frac{\mathrm{d} V}{\mathrm{~d} P}\right)+b_{2} \cdot \frac{\mathrm{d} Q}{\mathrm{~d} V} \cdot \frac{\mathrm{d} V}{\mathrm{~d} P}=0
\end{aligned}
$$

\section{表 2. 生产厂商产品质量与生产环保的组合}

\begin{tabular}{llll}
\hline & & \\
& 质量达标, 环保达标 & 质量优异, 环保达标 & 质量不达标, 环保达标 \\
\hline \multirow{2}{*}{ 环保 } & 质量达标, 环保优异 & 质量优异, 环保优异 & 质量不达标, 环保优异 \\
& 质量达标，环保不达标 & 质量优异，环保不达标 & 质量不达标，环保不达标 \\
\hline
\end{tabular}




$$
\begin{gathered}
\frac{\partial \pi}{\partial V}=P \cdot \frac{\mathrm{d} Q}{\mathrm{~d} V}-\left(\frac{\partial C}{\partial Q} \cdot \frac{\mathrm{d} Q}{\mathrm{~d} V}+\frac{\partial C}{\partial V}\right)+\left(\frac{\mathrm{d} b_{1}}{\mathrm{~d} V} \cdot Q+b_{1} \cdot \frac{\mathrm{d} Q}{\mathrm{~d} V}\right)+b_{2} \cdot \frac{\mathrm{d} Q}{\mathrm{~d} V}=0 \\
\frac{\partial \pi}{\partial T}=-\frac{\partial C}{\partial T}+\frac{\mathrm{d} b_{2}}{\mathrm{~d} T} \cdot Q=0
\end{gathered}
$$

联立求解(14)、(15)、(16)三式, 可以求出质量优异、环保优异厂商的最佳产品价格、最佳性价比(产 品质量水平)和最佳工艺水平。此外, 有: $\pi(L, K)=\left(P+b_{1}+b_{2}\right) \cdot f(L, K)-(w L+r K)$ 。我们也可以得到 公式(5), 即, 追求利润最大化的厂商可以得到最优的生产要素组合。问题得解。

\section{3. 质量达标, 环保不达标}

如果生产厂商质量达标但生产环保不达标, 国家将对其进行征税或加以处罚, 并勒令其停产和限期 整改, 只有当环保达标后才允许其继续生产。

生产厂商环保不达标的情况仍然是一个监督博弯问题。博弯的参与人为环保监督机关和生产厂商, 环保监督机关的纯战略选择是检查或不检查, 生产厂商的纯战略选择是不达标生产或达标生产。表 3 概 括了对应不同纯战略组合的支付矩阵。其中, $C_{G 2}$ 为环保监督机关的检查成本, $F_{2}$ 是征收的环境税或罚 款金额, $\pi_{l 2}$ 为厂商达标生产时的利润, $\pi_{u 3}$ 为厂商不达标生产被发现时的利润, $\pi_{u 4}$ 为厂商不达标生产未 被发现时的利润。设 $C_{E 2}$ 为生产厂商达标生产时, 相对于不达标生产时所增加的成本, 即采取环保措施 时的治理成本, 则有 $C_{E 2}=\pi_{u 4}-\pi_{12}$ 。当生产厂商环保不达标被发现时, 必须停产整改, 待环保达标后才 能继续生产, 但监督机关的处罚并不影响其产品的销售。设停产损失与整改费用之和(简称停产整改费用) 为 $C_{E 3}$, 则有 $\pi_{u 3}=\pi_{u 4}-C_{E 3}=\pi_{l 2}+C_{E 2}-C_{E 3}$ 。 $\pi_{l 2}$ 的表达式仍然如(5)式所示。

假定 $C_{G 2}<F_{2}$, 此时不存在纯战略纳什均衡, 但可以求解混合战略纳什均衡。设环保监督机关检查 的概率为 $\alpha$, 生产厂商违规生产销售的概率为 $\beta$ 。给定 $\beta$, 监督机关选择检查 $(\alpha=1)$ 和不检查 $(\alpha=0)$ 的期望收益分别为:

$$
\begin{gathered}
\pi_{G 2}(1, \beta)=\left(F_{2}-C_{G 2}\right) \beta+\left(-C_{G 2}\right)(1-\beta)=\beta F_{2}-C_{G 2} \\
\pi_{G 2}(0, \beta)=0 \beta+0(1-\beta)=0
\end{gathered}
$$

解 $\pi_{G 2}(1, \beta)=\pi_{G 2}(0, \beta)$, 得: $\beta^{*}=\frac{C_{G 2}}{F_{2}}$ 。即: 如果生产厂商不达标生产的概率小于 $C_{G 2} / F_{2}$, 环保' 监督机关的最优选择是不检查; 如果生产厂商不达标生产的概率大于 $C_{G 2} / F_{2}$, 环保监督机关的最优选择 是检查; 如果生产厂商不达标生产的概率等于 $C_{G 2} / F_{2}$, 环保监督机关随机地选择检查或不检查。

给定 $\alpha$, 生产厂商选择达标生产 $(\beta=0)$ 和不达标生产 $(\beta=1)$ 的期望收益分别为:

$$
\begin{gathered}
\pi_{E 2}(\alpha, 0)=\pi_{l 2} \alpha+\pi_{l 2}(1-\alpha)=\pi_{l 2} \\
\pi_{E 2}(\alpha, 1)=\left(\pi_{u 3}-F_{2}\right) \alpha+\pi_{u 4}(1-\alpha)=\left(\pi_{l 2}+C_{E 2}-C_{E 3}-F_{2}\right) \alpha+\left(\pi_{l 2}+C_{E 2}\right)(1-\alpha) \\
=\left(\pi_{l 2}+C_{E 2}\right)-\left(C_{E 3}+F_{2}\right) \alpha
\end{gathered}
$$

解 $\pi_{E 2}(\alpha, 0)=\pi_{E 2}(\alpha, 1)$, 得: $\alpha^{*}=\frac{C_{E 2}}{C_{E 3}+F_{2}}$ 。即: 如果监督机关检查的概率小于 $C_{E 2} /\left(C_{E 3}+F_{2}\right)$, 生产厂商的最优选择是不达标生产; 如果监督机关检查的概率大于 $C_{E 2} /\left(C_{E 3}+F_{2}\right)$, 生产厂商的最优选择 是达标生产; 如果监督机关检查的概率等于 $C_{E 2} /\left(C_{E 3}+F_{2}\right)$, 生产厂商随机地选择达标生产或不达标生产。

因此, 混合战略纳什均衡是: $\alpha^{*}=C_{E 2} /\left(C_{E 3}+F_{2}\right), \beta^{*}=C_{G 2} / F_{2}$, 即环保监督机关以 $C_{E 2} /\left(C_{E 3}+F_{2}\right)$ 的概率检查, 生产厂商以 $C_{G 2} / F_{2}$ 的概率选择不达标生产。这个均衡的另一个可能的(或许更为合理的)解 释是, 经济中有许多个生产厂商, 其中有 $C_{G 2} / F_{2}$ 比例的生产厂商选择不达标生产, $\left(1-C_{G 2} / F_{2}\right)$ 比例的 
生产厂商选择达标生产; 环保监督机关随机地检查 $C_{E 2} /\left(C_{E 3}+F_{2}\right)$ 比例的生产厂商的生产情况。

环保监督博弯的纳什均衡与监督机关的检查成本 $C_{G 2}$ 、治理成本 $C_{E 2}$ 、停产整改费用 $C_{E 3}$ 和所征收的 环境税或罚款金额 $F_{2}$ 有关。对不达标生产征税或惩罚越重、监督机关的检查成本越低, 厂商选择不达标 生产的概率越小; 对不达标生产征税或惩罚越轻、监督机关的检查成本越高, 厂商选择不达标生产销售 的概率越大。这个结论与我们关于生产厂商逃避环保检查的技术和检查成本的假设有关。我们假定一旦 环保监督机关检查, 不达标生产就会被发现。如果不是这样, 比如说, 如果违规严重而获利颇多的生产 厂商有更好的办法进行隐蔽生产, 从而使违规行为更难被发现, 这个结论就不一定成立了。此外, 违规 严重而获利颇多的生产厂商可能更有积极性贿赂监督官员, 在这种情况下, 上述结论也难以成立。但是 有一点可以肯定的是, 通过提高对违规者的惩罚, 生产厂商违规的积极性就会下降, 监督机关检查的必 要性也会降低。所以, 监督机关应努力改进检查技术, 降低检查成本, 并应制定科学的税收或罚款制度, 比如根据违规产品的销量和违规性质(生产工艺环保性能的优劣、有无造成严重的环境问题和不良影响等) 决定税额或罚款额度。一般情况下, 生产工艺环保性能越优、造成的环境问题和影响越小, 税额或罚款 越轻; 生产工艺环保性能越差、造成的环境问题和影响越大, 税额或罚款越重。这样, 违规性质越严重, 出现的可能性越小, 即严重违规厂商的数量和严重违规的次数越少。

下面详细分析一下 $\alpha^{*}=C_{E 2} /\left(C_{E 3}+F_{2}\right)$ 的意义。由于 $C_{E 2}>0, C_{E 3}>0, F_{2}>0$, 所以 $\alpha^{*}>0$ 。若监 督机关打算通过首次监督发现就彻底解决问题, 即 $\alpha^{*} \leq 1$, 则必须有 $F_{2} \geq C_{E 2}-C_{E 3}$; 若问题比较严重, 厂商的治理成本太高, 有 $C_{E 2}>C_{E 3}+F_{2}, \alpha^{*}>1$, 则需要通过多次监督发现才能彻底解决问题, 此时, 监督机关的累计罚款与生产厂商的累积停产整改费用之和必须不小于治理成本, 否则, 生产厂商不会达 标生产。一般情况下, 生产厂商违规性质越严重或治理难度越大, $C_{E 2}$ 的值就越大, 那么, 如果 $F_{2}$ 给定, 并设 $C_{E 3}$ 与 $C_{E 2}$ 成正比, 则违规性质越严重或治理难度越大, $\alpha^{*}$ 的值越大, 即监督机关需要对有严重违 规倾向和治理难度大的生产厂商加大检查力度。但是, 在 $C_{E 3}$ 与 $C_{E 2}$ 成正比的情况下, 如果设定 $F_{2}$ 的值 与 $C_{E 2}$ 的值成正比, 则不管违规性质和治理难度如何, $\alpha^{*}$ 的值可以基本保持不变, 有 $\alpha^{*} \cong \alpha_{0}^{*}$ 。所以, 如 果税额或罚款额度 $F_{2}$ 设置合理, 只要监督机关以 $\alpha_{0}^{*}$ 以上的概率检查所有生产厂商的生产情况, 或随机地 检查 $\alpha_{0}^{*}$ 以上比例生产厂商的生产情况, 则生产厂商选择不达标生产的期望收益将小于选择达标生产的期 望收益, 那么, 生产厂商在大多数情况下将选择达标生产, 或者说, 一般情况下, 大多数的生产厂商将 选择达标生产，结果理想时，则可以基本杜绝不达标生产情况的发生。 $F_{2}$ 的设置可以如下: $F_{2}=f_{2}(T) \cdot Q(V)$, 其中, $f_{2}(T)$ 为单位产品的税额或罚款金额, 是生产工艺环保水平 $T$ 的函数, 且与 $T$ 成反比例关系, $T$ 越高, 征税或罚款越轻, $T$ 越低, 征税或罚款越重; $Q(V)$ 为产品产量。不过, 对治理 难度确实很大的生产厂商, 征税或罚款 $F_{2}$ 也不能过重, 而只能在合理征税或罚款的同时加大检查力度。 当治理成本 $C_{E 2}$ 非常大时, 有 $\alpha^{*} \geq 1$, 即需要进行 $100 \%$ 甚至以上的检查。所以, 对治理成本非常大的生 产厂商, 有必要进行在线监测, 实时监督其生产环保情况。

就质量达标、环保不达标生产厂商的竞争力而言, 当监督机关的检查概率小于 $\alpha^{*}=C_{E 2} /\left(C_{E 3}+F_{2}\right)$ 时, 其竞争力强于环保达标生产厂商; 当监督机关的检查概率大于 $\alpha^{*}=C_{E 2} /\left(C_{E 3}+F_{2}\right)$ 时, 其竞争力弱于环 保达标生产厂商; 当监督机关的检查概率等于 $\alpha^{*}=C_{E 2} /\left(C_{E 3}+F_{2}\right)$ 时, 其竞争力与环保达标生产厂商的 竞争力相同。质量达标环保不达标生产厂商的违规行为虽然不会损害消费者的利益, 但有可能产生严重 的环境问题和恶劣的环境影响, 所以, 环保监督机关必须以大于 $\alpha^{*}=C_{E 2} /\left(C_{E 3}+F_{2}\right)$ 的概率对生产厂商 的生产情况进行检查, 以减少或杜绝不达标生产情况的发生。

由于没有积极意义, 在此我们不再求解质量达标环保不达标厂商的最佳劳动投入、最佳资本投入、 最佳产品价格、最佳产品质量。 


\section{4. 质量微观经济学的简单政策意义}

产品的性价比(价值系数, 质量水平)影响产品的价格、销量、使用成本和使用过程中的环境成本, 产 品的生产工艺影响产品生产过程中的环境成本, 为了控制产品的使用成本和环境成本, 政府可以通过标 准加税(对生产厂商的奖惩实际上就是一种税)的方式对产品质量和环境加以管理。随着标准的逐步完善和 日益严格, 产品的质量水平(包括环境性能)将会越来越高, 我国的环境状况也会越来越好。在这种管理模 式下, 产品的质量(包括性能)水平已成为影响厂商利润的最关键因素, 生产厂商便会想尽办法改进产品质 量、提高产品性能, 在产品的节能、降耗、减排和使用方便性、可靠性等指标上下功夫, 促进社会收益 的最大化。同时，政府还可以利用所征的税款投入环境治理中，进一步改善国家的环境状况。

\section{参考文献 (References)}

[1] 银路. 质量经济学概述 [J]. 电子质量, 1995(4): 22-25.

[2] 郭克莎. 质量经济学概论[M]. 广州: 广东人民出版社, 1992.

[3] 何晓晴. 质量经济学、质量经济性与质量经济辨析[J]. 企业家天地(理论版), 2007(7): 233-234.

[4] 石培忠, 谢承义, 编. 质量经济分析[M]. 贵州: 贵州人民出版社, 1987.

[5] 银路, 刘卫. 质量与效益的结合一谈质量经济分析的框架 [J]. 质量管理, 1990(5): 34-35.

[6] 深圳市质量发展战略课题组. “质量经济”分析一一劳动价值论深化研究中的一个新概念 [J]. 特区经济, 2002(5): 7-11.

[7] 高鸿业, 主编. 西方经济学[M]. 第二版. 北京: 中国人民大学出版社, 2000.

[8] 平狄克, 鲁宾费尔德, 著. 微观经济学[M]. 第四版. 张军, 等, 译. 北京: 中国人民大学出版社, 2000.

[9] 斯蒂格利茨, 著. 经济学(上册) [M]. 姚开建, 等, 译, 北京: 中国人民大学出版社, 1997.

[10] Vany, A.S. and Saving, T.R. (1983) The Economics of Quality. Journal of Political Economy, 91, 979-1000. http://dx.doi.org/10.1086/261196

[11] Sweeney, J. (1974) Quality, Commodity Hierarchies, and Housing Markets. Econometrica, 42, 147-168. http://dx.doi.org/10.2307/1913691

[12] Dreze, J.H. and Hagen, K.P. (1978) Choice of Product Quality: Equilibrium and Efficiency. Econometrica, 46, 493513. http://dx.doi.org/10.2307/1914227

[13] Nancy, R.L. (1990) Profitability and Product Quality: Economic Determinants of Airline Safety Performance. Journal of Political Economy, Part 1 October 1990, 98, 944-964.

[14] Riley, J. (1979) Informational Equilibrium. Econometrica, 47, 331-360. http://dx.doi.org/10.2307/1914187

[15] Plott, C.R. and Miller, R.M. (1985) Product Quality Signaling in Experimental Markets. Econometrica, 53, 837-872.

[16] Hassin, R. (1986) Consumer Information in Markets with Random Product Quality: The Case of Queues and Balking. Econometrica, 54, 1185-1196. http://dx.doi.org/10.2307/1912327

[17] Nevo, A. (2001) Measuring Market Power in the Ready-to-Eat Cereal Industry. Econometrica, 69, 307-342. http://dx.doi.org/10.1111/1468-0262.00194

[18] Goolsbee, A. and Petrin, A. (2004) The Consumer Gains from Direct Broadcast Satellites and the Competition with Cable TV. Econometrica, 72, 351-381. http://dx.doi.org/10.1111/j.1468-0262.2004.00494.x

[19] Jin, G.Z. and Kato, A. (2006) Price, Quality and Reputation: Evidence from an Online Field Experiment. Rand Journal of Economics, Forthcoming, Available at SSRN: http://ssrn.com/abstract=917315

[20] Kumar, P. (2006) Intertemporal Price-Quality Discrimination and the Coase Conjecture. Journal of Mathematical Economics, 42, 896-940. http://dx.doi.org/10.1016/j.jmateco.2006.04.013

[21] Biglaiser, G. and Ma, C.T.A. (2003) Price and Quality Competition under Adverse Selection: Market Organization and Efficiency. Rand Journal of Economics, 34, 56-71. http://dx.doi.org/10.2307/1593717

[22] Yohei, T. and Keita, K. (2013) Dynamic Voluntary Advertising and Vertical Product Quality. Economics Bulletin, 33, 564-574.

[23] Brad, K. (1998) Experience-Dependent Information Diffusion and Product Quality. Economic Inquiry, 36, 161-170. http://dx.doi.org/10.1111/j.1465-7295.1998.tb01703.x

[24] Kenneth, C.S. (2014) The Social Value of Information on Product Quality. Economics Letters, 122, 140-143. 
http://dx.doi.org/10.1016/j.econlet.2013.11.003

[25] Berndt, E.R., Bui, L.T., Reiley, D. and Urban, G.L. (1994) The Roles of Market, Product Quality and Price Competition in the Growth and Composition of the U.S. Anti-Ulcer Drug Industry. NBER Working Paper No. W4904. http://ssrn.com/abstract=226601

[26] Murphy, D. (2002) Price and Quality Relationships in Local Service Industries. FTC Bureau of Economics Working Paper No. 249. http://ssrn.com/abstract=387882

[27] Diehl, K., Kornish, L.J. and Lynch, J.G. (2003) Smart Agents: When Lower Search Costs for Quality Information Increase Price Sensitivity. Journal of Consumer Research, 30, 56-71. http://ssrn.com/abstract=340040 http://dx.doi.org/10.1086/374698

[28] 刘广第, 主编. 质量管理学[M]. 北京: 清华大学出版社, 1996.

[29] 张公绪, 主编. 新编质量管理学[M]. 北京: 高等教育出版社, 1998.

[30] 韩之俊, 章渭基, 编著. 质量工程学 [M]. 北京: 科学出版社, 1991.

[31] 吴礼民. 质量经济性的研究 [J]. 商业研究, 2003(23): 5-7.

[32] 刘宏. 企业质量经济性管理的研究[J]. 电子质量, 2006(10): 39-43.

[33] 银路. 不合格品率的经济性分析[J]. 电子质量, 1989(2): 10-12.

[34] 王㑆, 文昌俊. 基于过程能力的制造企业质量竞争力模型研究[J]. 中国机械工程, 2007, 18(14): 1691-1693.

[35] 魏国强. 企业质量经济分析的投入产出模型[J]. 中国纺织大学学报, 1995, 21(2): 129-135.

[36] 强加成, 徐景. 经济分析与成本管理在钢铁企业全面质量管理中的应用研究[J]. 河北冶金, 2005(4): 56-57.

[37] 朱朝晖. 广义质量成本及其特性曲线分析[J]. 商业研究, 2002(6): 41-42.

[38] 左春芳, 白宝光. 基于质量净收益的质量经济性分析[J]. 内蒙古工业大学学报(社会科学版), 2005, 14(2): 52-54.

[39] 黄培, 汪蓉. 基于质量净收益的质量经济性评价方法 [J]. 科学学与科学技术管理, 2002, 23(3): 55-57.

[40] 黄培, 汪蓉, 陈俊芳. 质量净收益与企业持续质量改进的经济性评价[J]. 工业工程与管理, 2002, 7(4): 50-53.

[41] 赵建华, 杨昌明, 童庆英. 最优质量水平——质量经济学的主要命题[J]. 科技进步与对策, 2005, 22(8): $186-187$.

[42] 王金德, 张东莉. 质量经济性——6SIGMA 管理的基本原则 $[\mathrm{J}]$. 上海质量, 2001(9): 20-22.

[43] 王金德, 丁胜, 张东莉. $6 \sigma$ 管理: 以经济性为原则的现代质量管理[J]. 工业工程与管理, 2002, 7(4): 35-40.

[44] 王金德. “隐蔽工程”开采的超额回报[J]. 上海质量, 2003(2): 18-20.

[45] 欧阳明德, 叶勇. $6 \sigma$ 管理法对现代质量管理的继承与发展[J]. 现代管理科学, 2004(5): 13-14.

[46] Bajic, V. (1988) Market Shares and Price-Quality Relationships: An Econometric Investigation of the U.S. Automobile Market. Southern Economic Journal, 54, 888-900. http://dx.doi.org/10.2307/1059524

[47] 张卓. 基于市场的最适宜质量水平模型[J]. 南京航空航天大学学报, 2001, 33(5): 474-477.

[48] 曾凤章, 崔丽. 基于顾客价值的质量经济性模型初探[J]. 北京理工大学学报(社会科学版), 2004, 6(5): 63-65.

[49] 陈江彪, 张卓. 基于顾客满意的产品质量经济性分析[J]. 南京理工大学学报(自然科学版), 2003, 27(S1): 38-42.

[50] 解文锋, 银路. 交货期的质量经济分析[J]. 电子质量, 1992(3): 26-30.

[51] 翟瑞华. 企业质量经济效益控制的实证研究[J]. 西安财经学院学报, 2008, 21(1): 89-91.

[52] 关晓光, 葛志杰. 质量经济效益的模糊综合评价[J]. 管理工程学报, 2000, 14(4): 65-68.

[53] 李少华. 质量经济效益分析[J]. 化工质量, 1999, 第 3, 4, 5, 6 期连载.

[54] 王兵才. 论质量经济学问题的研究框架[J]. 工业技术经济, 1991(4): 30-32.

[55] 李江帆. 质量经济学理论的开拓性研究——郭克莎著《质量经济学概论》评价[J]. 经济研究, 1992(7): 67-69(62).

[56] 张卓. 质量经济学发展评述[J]. 外国经济与管理, 2004, 26(9): 35-39.

[57] 多恩布什, 费希尔, 斯塔兹, 著. 宏观经济学[M]. 北京: 中国人民大学出版社, 2000.

[58] Romer, D. (2001) Advanced Macroeconomics. 2nd Edition, McGraw-Hill, New York.

[59] 庄子银, 著. 高级宏观经济学[M]. 武汉: 武汉大学出版社, 2004.

[60] Ball, B. (1995) Economic Growth. McGraw-Hill, New York.

[61] 谭崇台, 主编. 发展经济学[M]. 上海: 上海人民出版社, 1989. 
[62] 谭崇台, 主编. 发展经济学的新发展[M]. 武汉: 武汉大学出版社, 1999.

[63] 谭崇台, 主编. 发展经济学[M]. 太原: 山西经济出版社, 2001.

[64] Tordaro, M.P. (1994) Economic Development. Longman, London.

[65] 巴里·菲尔德, 玛莎·菲尔德, 著. 环境经济学[M]. 原毅军, 陈艳莹, 译. 北京: 中国财政经济出版社, 2006.

[66] Kohn, R.E. (1971) Optimal Air Quality Standards. Econometrica, 39, 983-995. http://dx.doi.org/10.2307/1909671

[67] 王学军. 环境质量经济价值度量的若干问题[J]. 管理世界, 1994(3): 193-197.

[68] 黄淑芹, 王丽. 从博弯论看环境保护[J]. 科技创业月刊, 2005, 18(12): 166.

[69] 胡品洁. 顾客导向和环境保护角度的质量经济分析 [J]. 商业研究, 2002(1): 49-51.

[70] 张维迎, 著. 博亦论与信息经济学[M]. 上海: 上海三联书店, 上海人民出版社, 1996. 